\title{
BMJ Open Cost-effectiveness of mechanical thrombectomy within 6 hours of acute ischaemic stroke in China
}

\author{
Yuesong Pan, ${ }^{1,2,3,4,5}$ Xueli Cai, ${ }^{6}$ Xiaochuan Huo, ${ }^{1,2,3,4}$ Xingquan Zhao, ${ }^{1,2,3,4}$ \\ Liping Liu, ${ }^{1,2,3,4}$ Yongjun Wang, ${ }^{1,2,3,4}$ Zhongrong Miao, ${ }^{1,2,3,4}$ Yilong Wang ${ }^{1,2,3,4}$
}

\begin{abstract}
To cite: Pan Y, Cai X, Huo $X$, et al. Costeffectiveness of mechanical thrombectomy within 6 hours of acute ischaemic stroke in China. BMJ Open 2018;8:e018951. doi:10.1136/ bmjopen-2017-018951

- Prepublication history and additional material for this paper are available online. To view these files, please visit the journal online (http://dx.doi. org/10.1136/bmjopen-2017018951).
\end{abstract}

YP and XC contributed equally.

Received 2 August 2017

Revised 29 December 2017

Accepted 17 January 2018

Check for updates

'Department of Neurology, Beijing Tiantan Hospital, Capital Medical University, Beijing,

China

${ }^{2}$ China National Clinical

Research Centre for

Neurological Diseases, Beijing,

China

${ }^{3}$ Centre of Stroke, Beijing Institute for Brain Disorders, Beijing, China

${ }^{4}$ Beijing Key Laboratory of Translational Medicine for Cerebrovascular Disease, Beijing, China

${ }^{5}$ Department of Epidemiology and Health Statistics, School of Public Health, Capital Medical University, Beijing, China

${ }^{6}$ Department of Neurology,

Lishui Hospital of Zhejiang

University (the Central Hospital

of Lishui), Lishui, China

Correspondence to

Dr. Zhongrong Miao;

zhongrongm@163.com and Dr

Yilong Wang;

yilong528@gmail.com

\section{ABSTRACT}

Objectives Endovascular mechanical thrombectomy is an effective but expensive therapy for acute ischaemic stroke with proximal anterior circulation occlusion. This study aimed to determine the cost-effectiveness of mechanical thrombectomy in China, which is the largest developing country.

Design A combination of decision tree and Markov model was developed. Outcome and cost data were derived from the published literature and claims database. The efficacy data were derived from the meta-analyses of nine trials. One-way and probabilistic sensitivity analyses were performed in order to assess the uncertainty of the results. Setting Hospitals in China.

Participants The patients with acute ischaemic stroke caused by proximal anterior circulation occlusion within 6 hours.

Interventions Mechanical thrombectomy within 6 hours with intravenous tissue plasminogen activator (tPA) treatment within 4.5 hours versus intravenous tPA treatment alone.

Outcome measures The benefit conferred by the treatment was assessed by estimating the cost per quality-adjusted life-year (QALY) gained in the long term (30 years).

Results The addition of mechanical thrombectomy to intravenous IPA treatment compared with standard treatment alone yielded a lifetime gain of 0.794 QALYS at an additional cost of CNY 50000 (US\$7700), resulting in a cost of CNY 63010 (US\$9690) per QALY gained. The probabilistic sensitivity analysis indicated that mechanical thrombectomy was cost-effective in $99.9 \%$ of the simulation runs at a willingness-to-pay threshold of CNY 125700 (US\$19 300) per QALY.

Conclusions Mechanical thrombectomy for acute ischaemic stroke caused by proximal anterior circulation occlusion within 6 hours was cost-effective in China. The data may be used as a reference with regard to medical resources allocation for stroke treatment in low-income and middle-income countries as well as in the remote areas in the developed countries.

\section{BACKGROUND}

Arterial recanalisation and subsequent reperfusion performed shortly after acute ischaemic stroke have demonstrated their ability to restore brain function. ${ }^{1}$ Besides

\section{Strengths and limitations of this study}

- A combination of decision tree and Markov model was developed in order to simulate the short-term and long-term costs and outcomes after mechanical thrombectomy for ischaemic stroke.

- The majority of the parameters used in the model, including costs, utilities and transition probabilities, were collected based on the Chinese setting, reflecting the situation in the low-income and middle-income countries.

- A limitation of the present study is that the health status and costs that resulted from additional causes other than stroke were not included in this model.

- An additional limitation is that the efficacy of the mechanical thrombectomy treatment was based on trials that were completed in high-income countries; however, these were the only data available.

intravenous recombinant tissue-type plasminogen activator (tPA) within 4.5 hours, endovascular mechanical thrombectomy is another effective reperfusion strategy, which can remove large, proximal clots rapidly and results in higher rate of reperfusion compared with intravenous tPA alone. ${ }^{2}$ Second-generation retrievable stents can achieve higher recanalisation rates compared with first-generation devices. Although trials that have used first-generation thrombectomy devices failed to demonstrate clinical benefit compared with intravenous tPA, the five recently published clinical trials in 2015 that included second-generation devices have shown clear benefits with regard to the addition of mechanical thrombectomy to standard treatment for acute ischaemic stroke caused by proximal anterior circulation occlusions. ${ }^{2-6}$

Although mechanical thrombectomy with second-generation devices exhibits optimal effectiveness with acceptable safety, the main disadvantage is the high cost that requires expensive devices, highly trained proceduralists and special periprocedural support. 


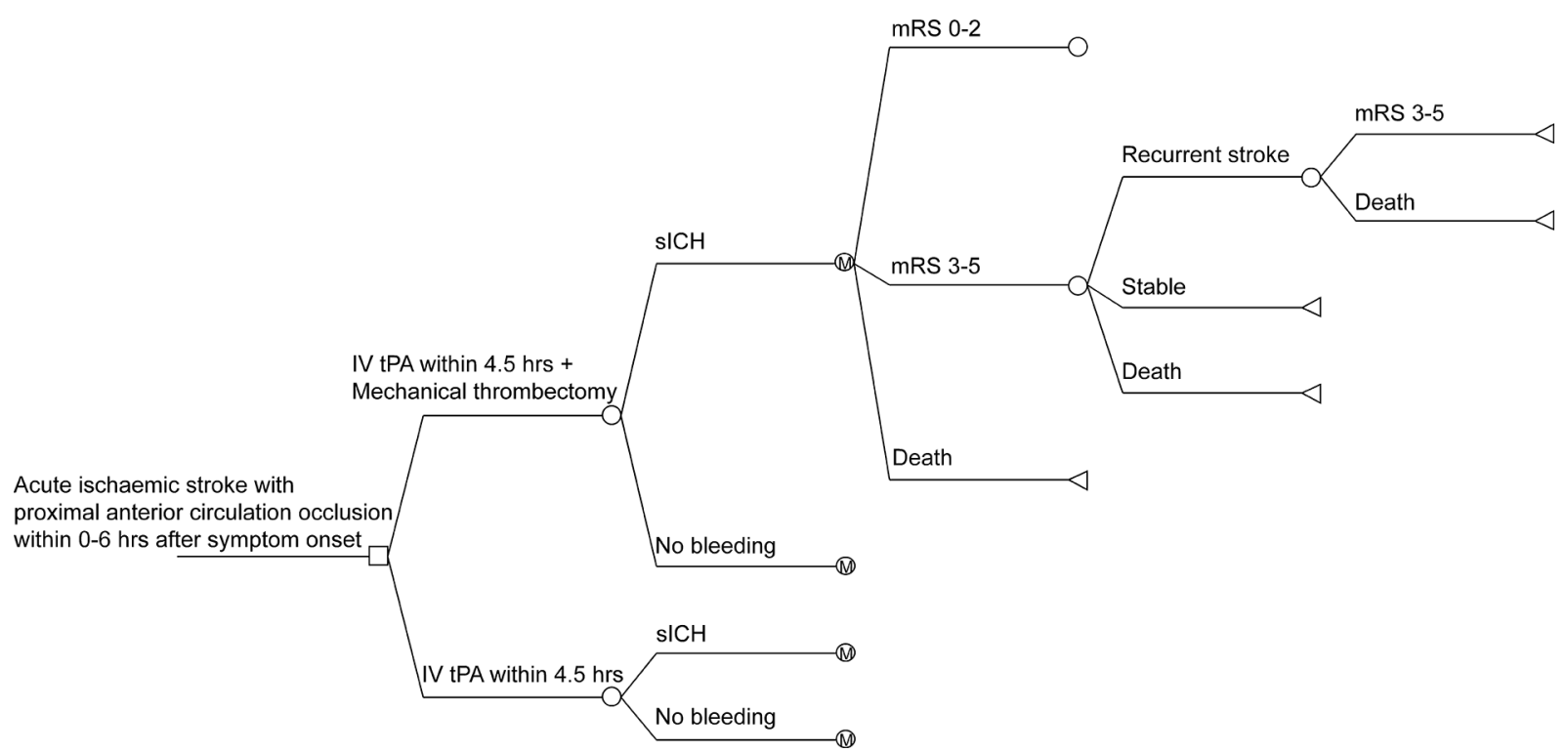

Figure 1 Decision tree and Markov state transition model. A patient with an acute ischaemic stroke with anterior circulation occlusion entered the model at 63 years old receiving either intravenous tissue-type plasminogen activator (tPA) with or without mechanical thrombectomy and transited between health states until death or 30 years. Patients may remain in the same health state, move to a state of equal or greater disability after recurrent stroke or die. Only transition from dependent state (modified Rankin Scale (mRS) 3-5) was illustrated in the figure. IV, intravenous; M, Markov node; sICH, symptomatic intracerebral haemorrhage.

Previous studies that have examined the economic aspects of this type of therapy were conducted in the USA, UK, Sweden, Canada and Spain. These studies indicated that mechanical thrombectomy treatment for acute ischaemic stroke was cost-effective or even cost-saving in the long term. ${ }^{7-15}$ However, all of these studies were conducted in high-income countries, and the corresponding results may not be applicable in low-income and middle-income countries such as China, where medical resources are scarce and stroke is a leading cause of death. ${ }^{16}$ Little is known with regard to the cost-effectiveness and feasibility of the application of mechanical thrombectomy treatment in patients with acute ischaemic stroke in low-income and middle-income countries. The analysis of the economic costs involved in mechanical thrombectomy in low-income and middle-income countries is urgent. In the present study, we aimed to evaluate the cost-effectiveness of the addition of mechanical thrombectomy treatment with second-generation devices to standard care for acute ischaemic stroke in the setting of China, which is considered the largest low-income and middle-income country.

\section{METHODS}

\section{Model overview}

A combination of decision tree and Markov model (figure 1) was developed in order to simulate the short-term (1, 5 and 6 years) and long-term (30 years) cost-effectiveness of mechanical thrombectomy using stent retrievers within 6 hours with intravenous tPA treatment within 4.5 hours versus intravenous tPA treatment alone within 4.5 hours after onset of stroke. The base case of the model was a cohort of 100000 patients with acute ischaemic stroke with proximal anterior circulation occlusion (36\% female), with mean age of 63 years old, being admitted to the hospital within 6 hours after onset of stroke. The baseline characteristics were the same as patients enrolled in the treated arm of the Endovascular therapy for Acute ischaemic Stroke Trial (EAST), a non-randomised interventional study that aimed to evaluate the safety and efficacy of Solitaire thrombectomy in Chinese patients with acute stroke) ${ }^{17}$ who were admitted to the hospital within 6 hours. Among the patients admitted to the hospital within 6 hours after the onset of stroke, we assumed that $85.4 \%$ patients were admitted within 4.5 hours in both arms according to the data from the China National Stroke Registry (CNSR); a nationwide registry that has recruited 21902 consecutive patients with acute cerebrovascular events from 132 hospitals in China between September 2007 and August $2008,{ }^{18}$ all of whom were assumed to receive intravenous tPA treatment, whereas all patients in the intervention arm received mechanical thrombectomy treatment. The total costs and quality-adjusted life-years (QALYs) that were gained with each alternative type of treatment were estimated for each health state at 90 days from the index event, at a 9-month period following this time point and then estimated annually for the remaining 30 years. The half-cycle correction was conducted for the years spent in the corresponding states that were subsequently used to calculate the health outcomes and costs. The transition probability and costs were discounted for the first 3-month segment and for the next 9-month segment in the first year. This analysis was conducted from the 
perspective of healthcare payers. The present study used published data and anonymised clinical data of patients from databases and therefore was exempt from institutional review board approval.

\section{Input parameters}

The model input parameters were drawn from the published literature and the claims database (table 1). The proportion of efficacy and safety outcomes at 90 days in the intravenous tPA group was estimated by meta-analysis based on a random effect model $\left(\mathrm{I}^{2}>50 \%\right)$ of the recently published nine trials, including the Multicentre Randomized Clinical Trial of Endovascular Treatment for Acute Ischaemic Stroke in the Netherlands, ${ }^{2}$ the Endovascular Treatment for Small Core and Anterior Circulation Proximal Occlusion with Emphasis on Minimizing CT to Recanalisation Times, ${ }^{3}$ the Randomized Trial of Revascularization with Solitaire FR Device versus Best Medical Therapy in the Treatment of Acute Stroke Due to Anterior Circulation Large Vessel Occlusion Presenting within Eight Hours of Symptom Onset ${ }^{4}$ the Extending the Time for Thrombolysis in Emergency Neurological Deficits-Intra-Arterial, ${ }^{5}$ the Solitaire with the Intention for Thrombectomy as Primary Endovascular Treatment (SWIFT PRIME), ${ }^{6}$ the THRombectomie des Artères CErebrales, ${ }^{19}$ The Randomized, Concurrent Controlled Trial to Assess the Penumbra System's Safety and Effectiveness in the Treatment of Acute Stroke, ${ }^{20}$ the Pragmatic Ischaemic Thrombectomy Evaluation ${ }^{21}$ and the Endovascular Acute Stroke Intervention. ${ }^{22}$ The efficacy and safety outcomes ORs of mechanical thrombectomy-treated patients at day 90 were estimated by meta-analysis based on a fixed effect model $\left(\mathrm{I}^{2}<50 \%\right)$ of the aforementioned nine trials (table 1). Subsequently, the proportions of the outcomes in the mechanical thrombectomy-treated groups were calculated based on the proportions in the intravenous tPA group and the ORs for the outcomes measured, as determined by the following formula: $\mathrm{p} 2=(\mathrm{OR} * \mathrm{p} 1) /$ $(1+(\mathrm{OR}-1) * \mathrm{p} 1)$.

The recurrent rates of stroke by modified Rankin Scale (mRS) categories and the death rate with recurrent strokes in years following the first 90 days were estimated from the CNSR study. ${ }^{18}$ We further assumed an increase in the risk of stroke recurrence by 1.03 -fold per life year. ${ }^{23}$ The patients who remained alive after stroke recurrence were assumed to be reallocated equally among categories of equal and greater disability. ${ }^{24}$ This indicated that patients in the independent state (mRS 0-2) who had a recurrent stroke and survived were allocated equally among the independent (mRS 0-2) and the dependent states (mRS 3-5), while patients in the dependent state remaining alive after stroke recurrence were all remain in dependent state.

The age-specific non-stroke death rates were derived from the most recent published census of China and were adjusted according to the causes of death of 2013 reported in the China Health Statistics Yearbook 2014. ${ }^{25} 26$ The disability status was assumed to affect the survival rate, and therefore the final age-specific non-stroke death rates for those with dependent state were adjusted by the HR of death for mRS $3-5 .{ }^{27}$

\section{Costs}

The total costs including both out-of-pocket costs and reimbursements were converted to 2013 Chinese yuan renminbi (CNY) using the medical care component of consumer price index. ${ }^{26}$ The average cost of one-time hospitalisation and annual posthospitalisation costs (such as inpatient and outpatient rehabilitation and secondary preventive costs) after stroke according to the categories of $\mathrm{mRS}$ were obtained from the database of the CNSR study. ${ }^{18}$ The additional costs of mechanical thrombectomy, including costs for devices, procedure and special periprocedural care, were estimated using the data from the EAST study and the Thrombolysis Implementation and Monitor of acute ischaemic Stroke in China (TIMS-CHINA; a national prospective registry of 1440 acute ischaemic stroke patients with thrombolytic therapy with intravenous tPA recruited from 67 centres in China between May 2007 and April 2012) study. ${ }^{17}$ The additional costs of tPA treatment and occurrence of sICH were estimated using the data from the CNSR and the TIMS-CHINA studies. We did not include the indirect economic costs such as lost work productivity in the present study. All costs and utilities were discounted by $3 \%$ per year. ${ }^{29}$

\section{Health states}

Patients could undergo transitions between the three health states according to the functional outcome based on mRS: independent (mRS 0-2), dependent (mRS 3-5) or deceased (mRS 6) ${ }^{730}$ At the end of each Markov cycle, the patients either remained in their current health state, attained a health state with greater disability due to recurrent stroke or did not survive due to a recurrent stroke or a non-stroke cause (figure 1).

\section{Outcome assessment}

Health outcomes were measured in terms of QALYs, which were calculated by multiplying the length of life by utility scores derived from the literature. ${ }^{9} 3132$ The utility scores of the different disability states after stroke were developed using the European quality of life scale European quality of life scale-5 dimensions (EQ-5D) along with the Chinese preference weights in a Chinese stroke population. ${ }^{32}$ In the current model, the events of recurrent stroke and sICH were considered temporary health states unless they resulted in death. All patients who entered these health states were assumed to have a short-term disutility of 30 days for an event of recurrent stroke and only 14 days for an event of sICH. ${ }^{931}$ The total economic costs were calculated by multiplying the number of patients in each state by the direct medical costs for that state. The incremental cost-effectiveness ratio (ICER) was calculated by dividing the difference of the costs by the difference in QALYs between the two 
Table 1 Base case and plausible ranges of model inputs

\begin{tabular}{|c|c|c|c|}
\hline Model input & Base case & Range & Reference \\
\hline Efficacy and safety outcome inputs & & & $2-619-22$ \\
\hline \multicolumn{4}{|l|}{ Proportion of outcomes at 90 days in intravenous tPA group } \\
\hline Death (mRS 6) & 0.168 & $0.131-0.205$ & \\
\hline $\mathrm{slCH}$ & 0.058 & $0.035-0.095$ & \\
\hline mRS 0-2 & 2.046 & $1.692-2.474$ & \\
\hline Death (mRS 6) & 0.871 & $0.684-1.109$ & \\
\hline $\mathrm{slCH}$ & 0.965 & $0.665-1.399$ & \\
\hline \multicolumn{4}{|l|}{ Probabilities inputs } \\
\hline Proportion of patients received mechanical thrombectomy & 0.861 & $0.839-0.883$ & 2-6 19-22 \\
\hline $\mathrm{mRS} 0-2$ & 0.1026 & $0.0961-0.1093$ & \\
\hline mRS 3-5 & 0.1418 & $0.1303-0.1534$ & \\
\hline Relative risk of stroke recurrence per life year & 1.03 & $1.02-1.04$ & 23 \\
\hline Death with recurrent stroke & 0.2101 & $0.1887-0.2316$ & CNSR \\
\hline Age specific non-stroke death rate* & $0.0089-0.1653$ & & 2526 \\
\hline HR of non-stroke death for mRS 3-5 & 1.78 & $1.43-2.14$ & 27 \\
\hline \multicolumn{4}{|l|}{ Cost inputs (2013 Chinese yuan renminbi) } \\
\hline Additional costs of mechanical thrombectomy & 60821 & $52314-70311$ & EAST \\
\hline Additional costs of intravenous tPA treatment & 11179 & $10555-11829$ & CNSR, TIMS-CHINA \\
\hline mRS 0-2 & 7385 & $7156-7619$ & \\
\hline $\mathrm{mRS} 3-5$ & 11350 & $10730-11996$ & \\
\hline \multicolumn{4}{|l|}{ Utility inputs } \\
\hline mRS 0-2 & 0.76 & $0.69-0.82$ & 32 \\
\hline $\mathrm{mRS} 3-5$ & 0.21 & $0.17-0.26$ & 32 \\
\hline Death (mRS 6) & 0 & $0.00-0.00$ & 32 \\
\hline Recurrent stroke & 0.34 & $0.32-0.36$ & 9 \\
\hline $\mathrm{sICH}$ & 0.84 & $0.72-1.0$ & 31 \\
\hline \multicolumn{4}{|l|}{ Discount rate inputs } \\
\hline Costs & 0.03 & $0.03-0.08$ & 29 \\
\hline Outcomes & 0.03 & $\pm 20 \%$ & 29 \\
\hline
\end{tabular}

All costs were converted to 2013 Chinese yuan renminbi by using the medical care component of consumer price index; to convert CNY to US\$, divide by 6.5 .

*Age-specific non-stroke death rate: only the number of 63 years old $(0.0089)$ and 93 years old $(0.1653)$ are presented.

CNSR, China National Stroke Registry; EAST, Endovascular therapy for Acute ischaemic Stroke Trial; mRS, modified Rankin Scale; sICH, symptomatic intracerebral haemorrhage; TIMS-CHINA, Thrombolysis Implementation and Monitor of acute ischaemic Stroke in China; tPA, tissue plasminogen activator. 
treatment alternatives. We modelled the costs and QALYs gained over the short-term (1, 5 and 6 years) and the long-term (30 years). The mechanical thrombectomy was considered cost-effective if the ICER was less than CNY 125700 (3× gross domestic product (GDP) per capita of China in 2013, ${ }^{26}$ US $\$ 19$ 300) per QALY gained. This willingness-to-pay threshold was recommended by the Commission on Macroeconomics and Health of WHO. ${ }^{29}$

\section{Sensitivity analysis}

A deterministic one-way sensitivity analysis that used varying probabilities, utilities and costs was conducted in order to evaluate the uncertainty of the long-term (30 years) results of the model. The variation in these parameters was conducted once at a time at the plausible rages (table 1). In order to determine how much worse mechanical thrombectomy could have performed but still produced a cost-effective ICER, we constructed the two hypothetical worse outcomes for the performance of the mechanical thrombectomy. This was accomplished by setting the ORs of mRS 0-2 at day 90 for the mechanical thrombectomy treatment at the lower limits of $90 \%$ and $95 \% \mathrm{CI}$ in the meta-analyses of the nine trials. This represented the unfavourable and worse unfavourable scenarios, respectively. For each outcome scenario, we further constructed four hypothetical cost scenarios by setting $10 \%$ increase or $10 \%, 25 \%$ and $50 \%$ decrease of the costs of mechanical thrombectomy.

Furthermore, a probabilistic sensitivity analysis was further undertaken in order to evaluate the stochastic uncertainty due to the simultaneous variability of the variables examined. It was conducted by using Monte Carlo simulation in Ersatz V.1.3 (a bootstrap add-in for Microsoft Excel for Windows; EpiGear International, Brisbane, Australia). We assumed that costs followed a log-normal distribution and that the probabilities and utilities followed a beta distribution. The simulation was run 10000 times. The results were summarised using a scatter plot and a cost-effectiveness acceptability curve.

\section{RESULTS}

\section{Base case analysis}

Table 2 indicates the costs, outcomes and ICER for the mechanical thrombectomy treatment calculated in the short term (1, 5 and 6 years) and in the long term (30 years). In the base case scenario, for a 63-year-old patient with acute ischaemic stroke caused by proximal anterior circulation occlusion within 6 hours after onset of stroke, mechanical thrombectomy would be cost-ineffective in the first 5 years, but become cost-effective from the sixth year onwards, using the threshold of CNY 125700 (3× GDP per capita of China in 2013, US $\$ 19300$ ) as the willingness-to-pay per QALY. After 6 years, the mechanical thrombectomy gained 0.430 QALYs at an additional cost of CNY 48940 (US\$7530), yielding an ICER of CNY 113800 (US\$17 510) per QALY gained. In the long term (30 years), mechanical thrombectomy gained 0.794 QALYs at an additional cost of CNY 50000 (US\$7700), yielding an ICER of CNY 63010 (US\$9690) per QALY gained.

\section{Sensitivity analysis}

The results of the deterministic one-way sensitivity analysis for the ICER of the mechanical thrombectomy in the long term are presented in the tornado diagrams (figure 2). Overall, the ICER was most sensitive to the OR of the favourable functional outcome (mRS 0-2) at day 90 , additional cost of mechanical thrombectomy and utility of mRS 0-2. In case of a decrease in the OR of mRS 0-2 at day 90 to 1.692, the ICER of the mechanical thrombectomy (CNY 87 123/QALY) was still within the threshold of the willingness-to-pay per QALY (CNY 125 700, 3× GDP per capita of China in 2013). In each hypothetical case scenario, the mechanical thrombectomy continued to produce a benefit in QALYs (online supplementary table 1). Even in the worst hypothetical scenario with worse unfavourable effect outcome and an increase of $10 \%$ price of the mechanical thrombectomy, this type

\begin{tabular}{|c|c|c|c|c|}
\hline Time horizon & Treat strategy & QALYs & Cost (CNY) & ICER (CNY/QALY) \\
\hline & Intravenous tPA alone & 0.326 & 27220 & - \\
\hline \multirow[t]{2}{*}{1 year } & $\begin{array}{l}\text { Mechanical thrombectomy+intravenous } \\
\text { tPA }\end{array}$ & 0.405 & 77700 & 638987 \\
\hline & Intravenous tPA alone & 1.392 & 58590 & - \\
\hline \multirow[t]{2}{*}{5 years } & $\begin{array}{l}\text { Mechanical thrombectomy+intravenous } \\
\text { tPA }\end{array}$ & 1.765 & 107710 & 131689 \\
\hline & Intravenous tPA alone & 1.599 & 65230 & - \\
\hline \multirow[t]{2}{*}{6 years } & $\begin{array}{l}\text { Mechanical thrombectomy+intravenous } \\
\text { tPA }\end{array}$ & 2.029 & 114170 & 113814 \\
\hline & Intravenous tPA alone & 2.979 & 117940 & - \\
\hline 30 years & $\begin{array}{l}\text { Mechanical thrombectomy+intravenous } \\
\text { tPA }\end{array}$ & 3.773 & 167970 & 63010 \\
\hline
\end{tabular}

ICER, incremental cost-effectiveness ratio; QALY, quality-adjusted life-year; tPA, tissue plasminogen activator. 
Odds ratio of mRS 0-2 at day 90,2.046(1.692-2.474)

Additional cost of mechanical thrombectomy, $¥ 60821$ ( $¥ 52314$ - $¥ 70311$ )

Utility of mRS 0-2,0.76(0.69-0.82)

Proportion of mRS 0-2 at 90 days in IV tPA group, $0.325(0.258-0.392)$

$\frac{\frac{10}{2}}{\frac{\pi}{\frac{10}{2}}}$

Utility of mRS 3-5, 0.21(0.17-0.26)

Costs discount rate, $0.03(0.03-0.08)$

Outcomes discount rate, $0.03(0.024-0.036)$

Recurrent rate of stroke (per patient year,mRS 0-2),0.1026(0.0961-0.1093)

Relative risk of stroke recurrence per life year,1.03(1.02-1.04)

Proportion of patients received mechanical thrombectomy, $0.861(0.839-0.883)$
$¥ 87,123$

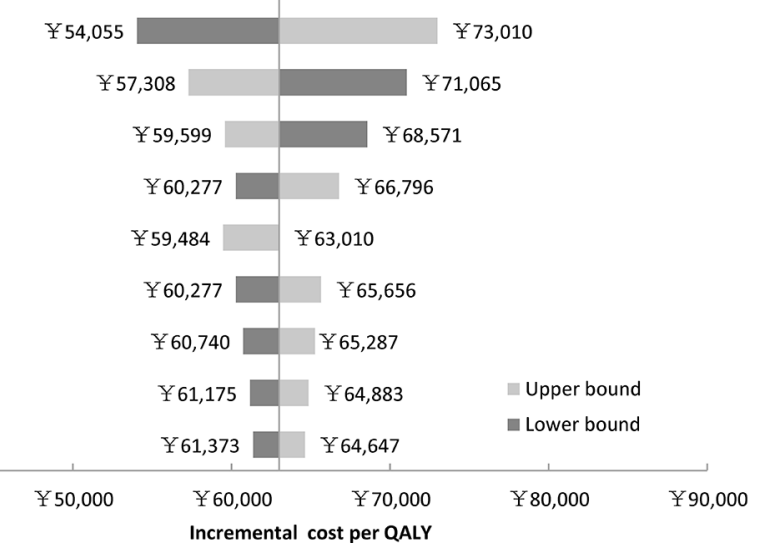

of treatment continued to be cost-effective (ICER: CNY $95839 /$ QALY). In the hypothetical case scenario with a base case effect outcome and a decrease of $50 \%$ in the price of mechanical thrombectomy, this type of treatment could be highly cost-effective (ICER: CNY 30 995/ QALY $<$ CNY 41900 [1× GDP per capita of China in 2013, US\$6400]/QALY).

Figure 3 indicates the results of the probabilistic sensitivity analysis in the long term with parameters of the model inputs presented in the online supplementary table 2. Among the 10000 simulation runs, mechanical thrombectomy was cost-effective in $99.9 \%$ of the simulations at a willingness-to-pay threshold of CNY 125700 (3× GDP per capita of China in 2013, US $\$ 19$ 300) per QALY. The online supplementary figure 1 indicates the

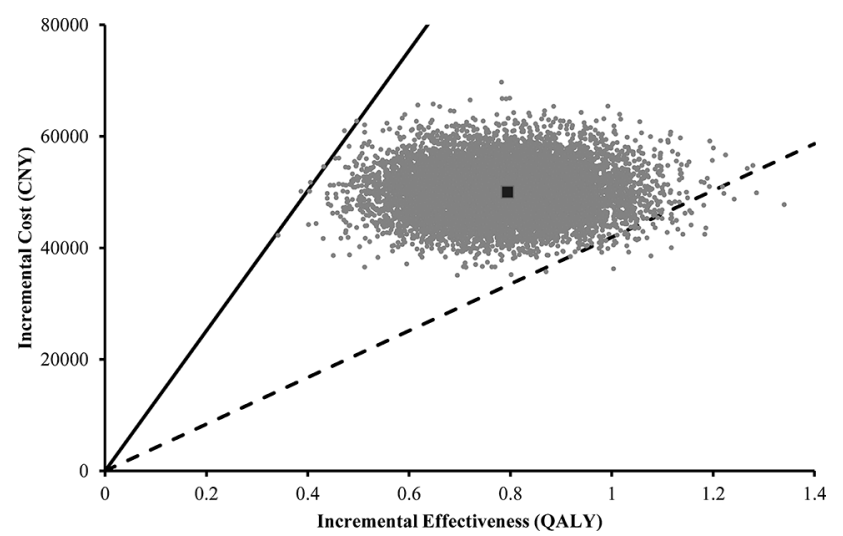

Figure 3 Scatter plot of the result of probabilistic sensitivity analysis in the long term (30 years). Each point represents a simulation. The dark square represents the base case $(0.794$ QALYs gained at an incremental cost of CNY 50 000). The solid line represents the willingness-to-pay threshold of CNY 125700 per QALY. The dashed line represents CNY 41900 per QALY. Points to the right of the solid line are considered cost-effective. CNY, Chinese yuan renminbi; QALYs, qualityadjusted life-years. cost-effectiveness acceptability curve of the mechanical thrombectomy.

\section{DISCUSSION}

The present study indicated that mechanical thrombectomy with second-generation devices for acute ischaemic stroke that was caused by proximal anterior circulation occlusion was cost-effective from the sixth year onwards in China. Each patient with acute ischaemic stroke treated with mechanical thrombectomy gained an ICER of CNY 63010 per QALY in the long term, which was below $3 \times$ GDP per capita of China in 2013 (CNY 125 700). The study further demonstrated that the ICER was more sensitive to the OR of favourable functional outcome at day 90 , additional cost of mechanical thrombectomy and the utility for patients with independence.

The present study indicated that mechanical thrombectomy treatment was cost-effective in low-income and middle-income countries such as China, which is a similar conclusion to that completed in other high-income countries. $^{7-10}$ The lifetime gain of 0.794 QALYs with regard to the mechanical thrombectomy treatment for acute ischaemic stroke in this study was comparable with that reported in the high-income countries (0.7 QALYs in the USA, ${ }^{7} 1.05$ QALYs in the UK ${ }^{9}$ and 0.99 QALYs in Sweden ${ }^{10}$ ). The QALYs gain of mechanical thrombectomy treatment was relatively higher compared with that of the majority of the other treatments for stroke. For example, the lifetime QALYs gain was 0.42 for intravenous tPA treatment for acute ischaemic stroke within 4.5 hours,${ }^{33}$ and 0.17 for clopidogrel for secondary prevention of stroke compared with aspirin. ${ }^{34}$ This may be due to the large magnitude of the effect of the mechanical thrombectomy (OR 2.046).

Mechanical thrombectomy treatment with second-generation devices has been accepted as the standard of care for patients with acute ischaemic stroke caused by proximal anterior circulation occlusions within 6 hours 
after symptom onset. ${ }^{35} 36$ However, economic costs of the mechanical thrombectomy treatment are extremely high, especially in low-income and middle-income countries such as China, where people experience a higher incidence of stroke and higher prevalence of intracranial atherosclerosis compared with the Western countries. ${ }^{37}$ The additional cost of the mechanical thrombectomy was approximately fivefold higher compared with that noted for the one-time hospitalisation without tPA treatment and mechanical thrombectomy in China. This difference between the two aforementioned parameters was only 1.5 -fold in the USA. ${ }^{7}$ Previous studies indicated socioeconomic disparities with regard to the utilisation of mechanical thrombectomy for acute ischaemic stroke and the patients with low income who were resident in remote areas exhibited lower rates of mechanical thrombectomy utilisation. ${ }^{38} 39$ The implementation of mechanical thrombectomy treatment was, to some extent, dependent on the cost-effectiveness of the technology, which is particularly significant for the clinical decision in the low-income and middle-income countries. The present study supported the implementation of the mechanical thrombectomy treatment after acute ischaemic stroke in clinical practice in the low-income and middle-income countries from the perspective of economics. The data may provide an important reference for the low income and/or remote areas in the Western countries.

Proximal large vessel atherosclerotic stenosis or occlusion accounts for $35 \%-40 \%$ of all acute ischaemic strokes, ${ }^{17}$ among which approximately $40 \%$ are admitted to the hospital within 6 hours in China. ${ }^{18}$ Therefore, approximately $14 \%-16 \%$ of patients with ischaemic stroke were eligible for mechanical thrombectomy and might benefit from this procedure. However, the realworld implementation of endovascular thrombectomy treatment in low-income and middle-income countries and areas may be restricted by the poor awareness of the public, poor infrastructure, inefficient systems, deficiency of specialists and the time points of patient entry to the hospital (within 6 hours), which may cause inequity for those who cannot receive the technology. ${ }^{10}$ Thus, the education that is targeted to the public, hospital administration and governmental agencies should be improved so that users can fully understand the benefit and cost-effectiveness of thrombectomy. ${ }^{40}$ Furthermore, the service system redesign is required to establish efficient care chains and workflow with coordination between neurointerventinalists and other departments. Additionally, high experience and skills are required to perform this advanced technology, while the ways of providing interventional treatment are non-unstandardised and diverse in low-income and middle-income countries such as China. ${ }^{40}$ Future studies should focus on the organisation of standardised training for performing thrombectomy in order to compensate for the deficiency of intervention specialists in these countries and areas.
The current study has several limitations that should be considered when interpreting the results. First, our model focused on the impact of mechanical thrombectomy treatment on acute ischaemic stroke, and the health status and costs that were involved as a result of other causes, such as occurrence of intracranial haemorrhage and myocardial infarction, were not included in this model. Second, the costs of transfer to the hospitals doing mechanical thrombectomy were not included in this analysis. Third, functional improvement after rehabilitation was not considered in the model due to the lack of available data on the efficacy of rehabilitation. However, organised rehabilitation after stroke in China is poor. ${ }^{41}{ }^{42}$ Fourth, the current study arbitrarily assumed to some extent that the patients with independent state remaining alive after stroke recurrence were equally reallocated in the categories of independent and dependent states. However, this is not unprecedented in the modelling of cost-effectiveness analysis for stroke. ${ }^{24}$ Finally, the efficacy of mechanical thrombectomy treatment was based on trials that were completed in high-income countries with older age of participants compared with those reported in China. In addition, the majority of the trials were terminated early and all were sponsored by the industry, which may have caused potential risk of bias, whereas some of the patients included in the trials did not receive intravenous tPA treatment. However, the current analysis included all data that were possibly available. These limitations would have led to underestimation or overestimation of the true cost-effectiveness of mechanical thrombectomy treatment in low-income and middle-income countries.

\section{CONCLUSIONS}

Mechanical thrombectomy with second-generation devices for acute ischaemic stroke caused by proximal anterior circulation occlusion within 6 hours after the onset of stroke was cost-effective in China. The current study supports the implementation of mechanical thrombectomy treatment after acute ischaemic stroke in clinical practice in low-income and middle-income countries and may also be a reference to the low income and/or remote areas in the developed countries. Additional medical resources that are related to mechanical thrombectomy should be allocated in these areas.

Contributors YP, YoW and YiW designed the study and drafted the manuscript. YP, $\mathrm{XC}$ and $\mathrm{XH}$ : collected the data, performed the literature search and constructed the decision tree. XZ, LL and ZM interpreted the data and revised the manuscript.

Funding This work was supported by grants from the Ministry of Science and Technology of the People's Republic of China (2015BAl12B04, 201 5BAl12B02, 2016YFC0901000, 2016YFC0901001 and 2016YFC0901002), grants from Beijing Municipal Science and Technology Commission (Z15110200390000 and Z151100003915117), and grants from Beijing Municipal Commission of Health and Family Planning (No. 2016-1-2041, SML20150502).

Competing interests None declared.

Patient consent Obtained.

Provenance and peer review Not commissioned; externally peer reviewed. 
Data sharing statement We, as the authors of this original research article, state that there are no additional, unpublished data available from this study.

Open Access This is an Open Access article distributed in accordance with the terms of the Creative Commons Attribution (CC BY 4.0) license, which permits others to distribute, remix, adapt and build upon this work, for commercial use, provided the original work is properly cited. See: http://creativecommons.org/ licenses/by/4.0/

(C) Article author(s) (or their employer(s) unless otherwise stated in the text of the article) 2018. All rights reserved. No commercial use is permitted unless otherwise expressly granted.

\section{REFERENCES}

1. Lees KR, Bluhmki E, von Kummer R, et al. Time to treatment with intravenous alteplase and outcome in stroke: an updated pooled analysis of ECASS, ATLANTIS, NINDS, and EPITHET trials. Lancet 2010;375:1695-703.

2. Berkhemer OA, Fransen PS, Beumer D, et al. A randomized trial of intraarterial treatment for acute ischemic stroke. $N$ Engl J Med 2015;372:11-20.

3. Goyal M, Demchuk AM, Menon BK, et al. Randomized assessment of rapid endovascular treatment of ischemic stroke. $N$ Engl $J$ Med 2015;372:1019-30.

4. Saver JL, Goyal M, Bonafe A, et al. Stent-retriever thrombectomy after intravenous t-PA vs. t-PA alone in stroke. $N$ Engl $J$ Med 2015;372:2285-95

5. Jovin TG, Chamorro A, Cobo E, et al. Thrombectomy within 8 hours after symptom onset in ischemic stroke. N Engl J Med 2015;372:2296-306.

6. Campbell BC, Mitchell PJ, Kleinig TJ, et al. Endovascular therapy for ischemic stroke with perfusion-imaging selection. N Engl J Med 2015;372:1009-18

7. Leppert MH, Campbell JD, Simpson JR, et al. Cost-effectiveness of intra-arterial treatment as an adjunct to intravenous tissuetype plasminogen activator for acute ischemic stroke. Stroke 2015;46:1870-6.

8. Kunz WG, Hunink MG, Sommer WH, et al. Cost-Effectiveness of Endovascular Stroke Therapy: A Patient Subgroup Analysis From a US Healthcare Perspective. Stroke 2016;47:2797-804.

9. Ganesalingam J, Pizzo E, Morris S, et al. Cost-utility analysis of mechanical thrombectomy using stent retrievers in acute ischemic stroke. Stroke 2015;46:2591-8.

10. Aronsson M, Persson J, Blomstrand C, et al. Cost-effectiveness of endovascular thrombectomy in patients with acute ischemic stroke. Neurology 2016;86:1053-9.

11. Lobotesis K, Veltkamp R, Carpenter IH, et al. Cost-effectiveness of stent-retriever thrombectomy in combination with IV t-PA compared with IV t-PA alone for acute ischemic stroke in the UK. J Med Econ 2016;19:785-94.

12. Steen Carlsson K, Andsberg G, Petersson J, et al. Long-term costeffectiveness of thrombectomy for acute ischaemic stroke in real life: An analysis based on data from the Swedish Stroke Register (Riksstroke). Int J Stroke 2017;12:802-14.

13. de Andrés-Nogales F, Álvarez M, de Miquel María Ángeles, et al. Cost-effectiveness of mechanical thrombectomy using stent retriever after intravenous tissue plasminogen activator compared with intravenous tissue plasminogen activator alone in the treatment of acute ischaemic stroke due to large vessel occlusion in Spain. European Stroke Journal 2017;2:272-84.

14. Shireman TI, Wang K, Saver JL, et al. Cost-effectiveness of solitaire stent retriever thrombectomy for acute ischemic stroke: results from the SWIFT-PRIME Trial (Solitaire With the Intention for Thrombectomy as Primary Endovascular Treatment for Acute Ischemic Stroke). Stroke 2017;48:379-87.

15. Xie X, Lambrinos A, Chan B, et al. Mechanical thrombectomy in patients with acute ischemic stroke: a cost-utility analysis. CMAJ Open 2016;4:E316-25.

16. Yang G, Wang Y, Zeng Y, et al. Rapid health transition in China, 19902010: findings from the Global Burden of Disease Study 2010. Lancet 2013;381:1987-2015.

17. Miao Z, Huo X, Gao F, et al. Endovascular therapy for Acute ischemic Stroke Trial (EAST): study protocol for a prospective, multicentre control trial in China. Stroke Vasc Neurol 2016;1:44-51.

18. Wang Y, Cui L, Ji X, et al. The China National Stroke Registry for patients with acute cerebrovascular events: design, rationale, and baseline patient characteristics. Int J Stroke 2011;6:355-61.
19. Bracard S, Ducrocq X, Mas JL, et al. Mechanical thrombectomy after intravenous alteplase versus alteplase alone after stroke (THRACE): a randomised controlled trial. Lancet Neurol 2016;15:1138-47.

20. Mocco J, Zaidat OO, von Kummer R, et al. Aspiration thrombectomy after intravenous alteplase versus intravenous alteplase alone. Stroke 2016;47:2331-8.

21. Muir KW, Ford GA, Messow CM, et al. Endovascular therapy for acute ischaemic stroke: the Pragmatic Ischaemic Stroke Thrombectomy Evaluation (PISTE) randomised, controlled trial. J Neurol Neurosurg Psychiatry 2017;88:38-44.

22. Khoury NN, Darsaut TE, Ghostine J, et al. Endovascular thrombectomy and medical therapy versus medical therapy alone in acute stroke: A randomized care trial. $J$ Neuroradio 2017;44:198-202.

23. Pennlert J, Eriksson M, Carlberg B, et al. Long-term risk and predictors of recurrent stroke beyond the acute phase. Stroke 2014;45:1839-41.

24. Tung CE, Win SS, Lansberg MG. Cost-effectiveness of tissue-type plasminogen activator in the 3- to 4.5-hour time window for acute ischemic stroke. Stroke 2011;42:2257-62.

25. National Bureau of Statistics of China. The 2010 population census of the peaple's republic of china. http://www.stats.gov.cn/tjsj/pcsj/ rkpc/6rp/indexch.htm (accessed 25 Feb 2016).

26. Ministry of Health of the People's Republic of China. China health statistics yearbook 2014. Beijing: Peking Union Medical College Press, 2014.

27. Samsa GP, Reutter RA, Parmigiani G, et al. Performing costeffectiveness analysis by integrating randomized trial data with a comprehensive decision model: application to treatment of acute ischemic stroke. J Clin Epidemiol 1999;52:259-71.

28. Liao XL, Wang CX, Wang YL, et al. Implementation and outcome of thrombolysis with alteplase 3 to $4.5 \mathrm{~h}$ after acute stroke in Chinese patients. CNS Neurosci Ther 2013;19:43-7.

29. Pan Y, Wang A, Liu G, et al. Cost-effectiveness of clopidogrel-aspirin versus aspirin alone for acute transient ischemic attack and minor stroke. J Am Heart Assoc 2014;3:e000912.

30. Kim AS, Nguyen-Huynh M, Johnston SC. A cost-utility analysis of mechanical thrombectomy as an adjunct to intravenous tissue-type plasminogen activator for acute large-vessel ischemic stroke. Stroke 2011:42:2013-8.

31. Lee S, Anglade MW, Pham D, et al. Cost-effectiveness of rivaroxaban compared to warfarin for stroke prevention in atrial fibrillation. Am J Cardiol 2012;110:845-51

32. Wang YL, Pan YS, Zhao XQ, et al. Recurrent stroke was associated with poor quality of life in patients with transient ischemic attack or minor stroke: finding from the CHANCE trial. CNS Neurosci Ther 2014;20:1029-35.

33. Pan $\mathrm{Y}, \mathrm{Chen} \mathrm{Q}$, Zhao $\mathrm{X}$, et al. Cost-effectiveness of thrombolysis within 4.5 hours of acute ischemic stroke in China. PLoS One 2014:9:e110525.

34. Schleinitz MD, Weiss JP, Owens DK. Clopidogrel versus aspirin for secondary prophylaxis of vascular events: a cost-effectiveness analysis. Am J Med 2004;116:797-806.

35. Powers WJ, Derdeyn CP, Biller J, et al. 2015 American Heart Association/American Stroke Association Focused Update of the 2013 Guidelines for the Early Management of Patients With Acute Ischemic Stroke Regarding Endovascular Treatment: A Guideline for Healthcare Professionals From the American Heart Association/ American Stroke Association. Stroke 2015;46:3020-35.

36. Miao Z, Wang Y. Chinese guidelines for endovascular treatment for acute ischemic stroke 2015. Chin J Stroke 2015;10:590-606.

37. Wang Y, Zhao X, Liu L, et al. Prevalence and outcomes of symptomatic intracranial large artery stenoses and occlusions in China: the Chinese Intracranial Atherosclerosis (CICAS) Study. Stroke 2014;45:663-9.

38. Brinjikji W, Rabinstein AA, Cloft HJ. Socioeconomic disparities in the utilization of mechanical thrombectomy for acute ischemic stroke. $J$ Stroke Cerebrovasc Dis 2014;23:979-84.

39. Pérez de la Ossa N, Abilleira S, Dorado L, et al. Access to endovascular treatment in remote areas: analysis of the reperfusion treatment registry of catalonia. Stroke 2016;47:1381-4.

40. Miao Z. New era of stroke therapy and new challenges. Stroke Vasc Neurol 2016;1:6-7.

41. Ad L, Huang ZQ, Liu HT, et al. Attitude and behavior for stroke and rehabilitation in stroke patients and relatives. in Chinese). Chin $J$ Rehabil Theory Pract 2009;15:252-4.

42. Gao YL, Chen LD, Tao J, et al. Investigation on the investment and popularization of rehabilitation from cerebral apoplexy: in 2 communities of Xiamen City. Chin J Convalescent Med 2009;18:472-4. 